\title{
ARTíCULO \\ Distribución y concentración de Zn total en sedimentos del fiordo Aysén, sur de Chile, posterior al terremoto y tsunami de 2007
}

Distribution and concentration of total $\mathrm{Zn}$ in marine sediments in Aysén Fjord, southern Chile, after earthquake and tsunami 2007

\begin{abstract}
Ramón Ahumada1, Mauricio Garrido' ${ }^{1}$, Elizabeth Gonzalez ${ }^{1}$ y Anny Rudolph ${ }^{1}$
'Facultad de Ciencias, Universidad Católica de la Santísima Concepción, Alonso de Ribera 2850, CP: 4090541, Concepción, Chile.rahuma@ucsc.cl

Abstract.- Between February and May 2007, more than 1,200 seismic movement of intensity above 3 degrees on the Richter scale (i.e., swarming) and a 6.2 degree earthquake, occurred in Aysén Fjord, southern Chile. The epicenter was located at Liquiñe - Ofqui fault, which is perpendicular to the middle of the fjord. This resulted in a landslide of soils and rocks along the coastline and followed by a local tsunami towards the fjord head. As a consequence of this seismic activity, the possible suspension and redistribution of the total $\mathrm{Zn}$ in marine sediments Aysén Fjord, is analyzed. Sampling was carried out during the $13^{\circ}$ Campaign of the program Research Cruises on Marine Remote Areas: CIMAR13-FJORD, November 2007. The sampling stations chosen were same ones used in previous 1995 and 2001 cruises. Acid extracts of the samples were analyzed for $\mathrm{Zn}$, in quintuplicate replicas by atomic absorption spectrophotometer. The results showed large variability in the concentration of $\mathrm{Zn}$ among stations, but no significant difference was observed in the concentrations of total $\mathrm{Zn}$ between sampling sites. This suggests that resuspension and redistribution of contents total $\mathrm{Zn}$ in the sediments was a local event, which in turns affected only the middle of the fjord where the landslides of soil and rocks occurred.
\end{abstract}

Key words: Total zinc, suspension, redistribution, sediments, fjords, seismic movements, tsunami, Chile

\begin{abstract}
Resumen.- Entre febrero y mayo de 2007, se produjeron más de 1.200 movimientos sísmicos de una intensidad mayor de 3 grados Richter (i.e., enjambre) y un sismo de 6,2 grados, en el Fiordo Aysén, sur de Chile. El epicentro fue la Falla LiquiñeOfqui, ubicada perpendicular a la parte media del fiordo. Como resultado se produjo una avalancha de tierra y rocas en la costa y ondas de tsunami local, hacia la cabeza. Se analiza la posible suspensión y redistribución de Zn total en los sedimentos marinos del Fiordo Aysén, por efecto de la actividad sísmica ocurrida en el área. El muestreo se realizó durante la $13^{\circ}$ campaña del Programa Cruceros de Investigación Marina en Áreas Remotas, (CIMAR13-FIORDO), en noviembre de 2007. Las estaciones muestreadas fueron las mismas elegidas en campañas anteriores, 1995 y 2001. Se analizaron extractos ácidos de las muestras por quintuplicado, en un espectrofotómetro de absorción atómica. Los resultados mostraron una alta variabilidad en la concentración de Zn entre las estaciones. Sin embargo, no se observaron diferencias significativas en las concentraciones de Zn total entre los sitios de muestreo, lo que sugeriría que la resuspensión y redistribución de los contenidos de $\mathrm{Zn}$ total en los sedimentos fue un proceso local leve, que sólo afectó a la parte media del fiordo en que se produjo avalanchas de tierra y piedras.
\end{abstract}

Palabras clave: Zinc total, suspensión, redistribución, sedimentos, fiordos, sismicidad, tsunami, Chile

\section{INTRODUCCIÓN}

Los fiordos del sur de Chile son sistemas costeros semicerrados, con orientación este-oeste resultantes de la erosión glaciar. Se encuentran conectados a los valles longitudinales existentes, que a su vez los conectan a la zona costera (Clapperton 1994). Las cuencas producidas por la erosión glaciar, han sido modificadas por depósitos aluviales y coluviales y en menor proporción por sedimentos fluvio-glaciales recientes, resultantes de la degradación de rocas intrusivas plutónicas, lavas basálticas y de rocas ultra básicas, propias de la región (SERNAGEOMIN 2002'1, Delgado 2004).

${ }^{1}$ SERNAGEOMIN. 2002. Mapa Geológico de Chile. Serie Carta Geológica de Chile, № 75, Escala 1:1.000.000. SERNAGEOMIN, Santiago. 
Antezana (1999) a propósito de las comunidades bentónicas observadas en los fiordos, informa la existencia de una diversidad faunística propia en las diferentes subcuencas y menciona diferencias en sus respuestas a eventuales perturbaciones antropogénicas, situación que da cuenta de estructuras sedimentarias diferentes, que debieran ser consideradas al analizar el ingreso y la distribución de posibles contaminantes.

El Fiordo Aysén ( $\left.45^{\circ} 20^{\prime} \mathrm{S} ; 7^{\circ} 05^{\prime} \mathrm{W}\right)$, se ubica en la XI Región y forma parte del sistema de fiordos y canales del sur de Chile. La densidad poblacional en el área se estima en 0,8 habitantes por $\mathrm{km}^{2}$, con baja actividad antrópica. Posee $75 \mathrm{~km}$ de longitud y un basamento rocoso formado por rocas ígneas y metamórficas. Presenta desniveles con umbrales y cubetas o subcuencas, producidas por el avance y retroceso de las morrenas de los glaciales (Araya-Vergara 1997). Se encuentra formado por 3 subcuencas: una somera en la cabeza (ca., $100 \mathrm{~m}$ de profundidad), otra en la zona media del fiordo ( $c a$., $250 \mathrm{~m}$ profundidad) seguida de una de menor profundidad en la boca (ca., $180 \mathrm{~m})$, generadas por la acumulación de sedimentos de la morrena. La dinámica del fiordo presenta un régimen estuarino, clasificado como altamente estratificado (Pickard 1971, Silva et al. 1997, Silva \&
Guzmán 2006), que se conecta indirectamente a la costa sureste del océano Pacífico a través del Canal Moraleda. Recibe aguas residuales de la actividad industrial de Puerto Chacabuco y aguas continentales de los ríos Cuervos, Cóndor, Mañiguales y Aysén (Fig. 1). El tiempo de residencia de sus aguas se ha estimado en 6 meses (Salamanca 1996).

Estudios realizados en los sedimentos del Fiordo Aysén (Ahumada 1998, Ahumada et al. 2004), informaron áreas de mayor concentración de $\mathrm{Zn}$ total en la cabeza del fiordo en que se ubica Puerto Chacabuco, con un gradiente negativo en la concentración de $\mathrm{Zn}$ total hacia la boca, i.e., cabeza del fiordo con $181 \pm 29 \mu \mathrm{g} \mathrm{g}^{-1}$ (Ahumada 1998) y $168 \pm 15 \mu \mathrm{g} \mathrm{g}^{-1}$ (Ahumada et al. 2004), y boca con $85,8 \pm$ $4 \mu \mathrm{g} \mathrm{g}^{-1}$ (Ahumada 1998) y 83,6 $\pm 6 \mu \mathrm{g} \mathrm{g}^{-1}$ (Ahumada et al. 2004). El enriquecimiento observado en la cabeza del fiordo se asocia a la faena minera de embarque de concentrado de $\mathrm{Zn}$, que se realiza en Puerto Chacabuco. En los sedimentos del fiordo se estableció un intervalo como línea base de $\mathrm{Zn}$ total entre 84 y $86 \mu \mathrm{g} \mathrm{g}^{-1} \mathrm{de}$ sedimento seco, a través de las técnicas de análisis fraccionado de testigos y de especiación de $\mathrm{Zn}$ en los sedimentos (Ahumada 1998, Ahumada et al. 2004).

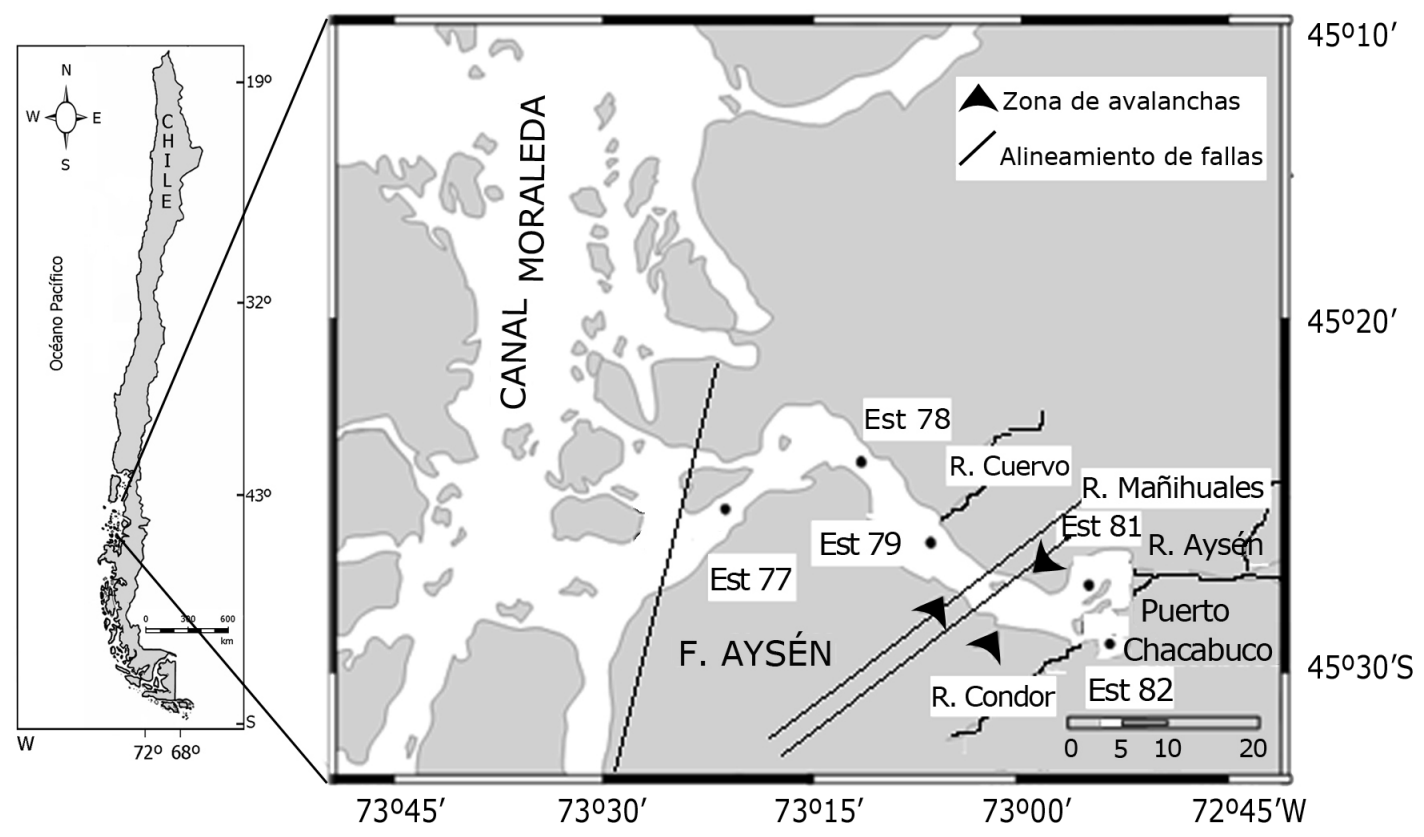

Figura 1. Ubicación de las estaciones muestreadas durante crucero CIMAR 13-FIORDO en el fiordo Aysén, Chile. Noviembre 2007. (Líneas indican alineamiento Falla Liqueñe-Ofqui) / Location of station sampled during the oceanographic cruise CIMAR 13-FJORD, at Aysén Fjord, Chile. November 2007. (Lines indicate alignment Liquiñe Ofqui fault) 
En el Fiordo Aysén, el 23 febrero 2007 se iniciaron numerosos eventos de actividad sísmica, generados por la activación de la Falla Liquiñe-Ofqui, con un alto porcentaje de movimientos sísmicos de intensidades mayores de 3 grados Richter. Durante febrero-mayo de 2007 se registró en el área más de 1200 sismos. La mayor intensidad se alcanzó el 21 de abril, cuando se produjo un sismo superficial cortical (Mw 6,2) que generó varias inestabilidades en las laderas rocosas y escarpadas del fiordo, con deslizamiento y avalanchas de tierra y rocas en varios sectores medios de ambas riberas del fiordo (Marín et al. 2008, 2010²). El impacto de las masas removidas de rocas en las aguas y su desplazamiento generaron ondas de tsunami local (Sepúlveda \& Serey 2009).

El objetivo de este trabajo fue analizar la posible suspensión y redistribución de Zn total en los sedimentos marinos del Fiordo Aysén, sur de Chile, por efecto de la actividad sísmica y tsunami ocurrido en el área en el 2007.

\section{Materiales y MÉTODOS}

Se utilizó como referencia para la planificación del muestreo y posterior análisis de resultados, la información de las campañas CIMAR 1-FIORDO 1995 (Ahumada 1998) y CIMAR 7-FIORDO 2001 (Ahumada et al. 2004, 2007).

El muestreo en el Fiordo Aysén se realizó en noviembre de 2007, como parte de la campaña CIMAR13-FIORDO, a bordo del buque 'AGOR Vidal Gormaz', coordinado por el Comité Oceanográfico Nacional (CONA-Chile). Con el objeto de comparar la información, la ubicación de las estaciones de muestreo en éste estudio, fueron las mismas 5 con que se trabajó en el crucero CIMAR 1-FIORDOS (1995) (Fig. 1).

Las muestras de sedimento fueron obtenidas mediante un box corer de 30 x 30 x $40 \mathrm{~cm}$. Los sedimentos en el box fueron sub-muestreados con testigos de PVC de $40 \mathrm{~cm}$, sellados, rotulados y guardados a bordo, a $4^{\circ} \mathrm{C}$. Del box corer se obtuvo además, muestras de sedimento superficial (primeros $4 \mathrm{~cm}$ ), las que fueron guardadas en bolsas de polietileno debidamente rotuladas, con el objeto de analizar granulometría y materia orgánica total, ésta última determinada gravimétricamente por la técnica de pérdida de peso por ignición (Luczak et al. 1997).

En el laboratorio se procedió a fraccionar por estratos los testigos de sedimento. Se utilizó para ello una espátula de teflón y los estratos de profundidad fueron: 0-2; 4-5; $6-7 ; 10-11 ; 15-16 ; 20-21 ; 30-31$ y 36-38 cm, dependiendo de la longitud del testigo. Cada sub-muestra de sedimento fraccionada fue secada a $60^{\circ} \mathrm{C}$ hasta peso constante, disgregada en un mortero de ágata y guardada rotulada en bolsas de polietileno de alta densidad, hasta su análisis.

Se sometieron $0,5 \mathrm{~g}$ de sedimento seco a digestión ácida [mezcla $5 \mathrm{ml} \mathrm{HNO}_{3}, 10 \mathrm{ml} \mathrm{HF} \mathrm{y} 7 \mathrm{ml} \mathrm{HClO}_{4}$ (Suprapur, Merck)], en vasos de teflón a temperatura constante $\left(70^{\circ} \mathrm{C}\right)$, hasta sequedad. El residuo fue disuelto en $10 \mathrm{ml}$ $\mathrm{HCl}$ Suprapur y aforado a $25 \mathrm{ml}$ con agua MilliQ. Se trabajó con 5 réplicas por estación.

Los extractos ácidos del sedimento fueron analizados en un espectrofotómetro de absorción atómica marca GBC Scientific Equipment, modelo 902, con tubo en la llama, con la técnica de adición estándar. La calibración del equipo se realizó con patrones de Zn y Li preparados a partir de un TitrisolMerc ${ }^{\circledR}$. La incerteza analítica o error analítico, fue estimado con material de referencia certificado para sedimentos (MESS-3, National Research Council Canada 2000) ${ }^{3}$. El porcentaje analítico de error respecto del material de referencia certificado fue mayor para el Zn i.e., 8,5\% (Tabla 1).

Tabla 1. Error analítico estimado utilizando material de referencia certificado MESS-3, (National Research Council of Canada, 2000) para los metales $\mathrm{Zn}$ y Li, utilizando el espectrofotómetro de absorción atómica, GBC $\mathbf{9 0 2}$ / Analytical error estimated from using Certified Reference Material MESS-3 (National Research Council of Canada, 2000) for $\mathrm{Zn}$ and Li metals, by Atomic Absorption Spectrophotometer, GBC 902

\begin{tabular}{|c|c|c|c|}
\hline \multirow{2}{*}{ Metal } & \multicolumn{2}{|c|}{ Incerteza o Error analítico } & \multirow{2}{*}{$\begin{array}{c}\text { Error } \\
\%\end{array}$} \\
\hline & $\begin{array}{l}\text { Experimental } \\
\left(\mu \mathrm{g} \mathrm{g}^{-1}\right)\end{array}$ & $\begin{array}{l}\text { MESS-3 } \\
\left(\mu \mathrm{g} \mathrm{g}^{-1}\right)\end{array}$ & \\
\hline $\mathrm{Li}$ & $77,2 \pm 4,3$ & $73,6 \pm 5,2$ & 4,5 \\
\hline $\mathrm{Zn}$ & $186,7 \pm 19,6$ & $172,0 \pm 16$ & 8,5 \\
\hline
\end{tabular}

${ }^{2}$ Marín VH, LE Delgado \& A Tironi. 2010. El tsunami del fiordo Aysén del 21 de abril de 2007: de las remociones en masa a la sociedad austral. Libro de Resúmenes, Simposio, XXX Congreso Anual de Ciencias del Mar, Concepción, Chile, p. 15.

${ }^{3}$ National Research Council Canada. 2000. MESS-3. Marine sediment reference materials for trace metals and other constituents. Measurement Science and Standards 1200 Montreal Road, Building M-12 Ottawa, Ontario K1A 0R6. Canada. <http://www.nrc-cnrc.gc.ca> 
Se determinó el factor de enriquecimiento (EF) para $\mathrm{Zn}$ $(\mathrm{M}+)$ partir de las concentraciones experimentales, utilizando al Li como normalizador (Aloupi \& Angelidis 2001) y la relación propuesta por Müller (1969), EF = [M+/ $\mathrm{Li}]$ / [M+/Li]), donde en el numerador [M+/Li] se utilizan las concentraciones experimentales y en el denominador $[\mathrm{M}+/ \mathrm{Li}]$, las concentración de línea base $\left(\mathrm{Zn}=84 \mu \mathrm{g} \mathrm{g}^{-1} \mathrm{y}\right.$ $\mathrm{Li}=26 \mu \mathrm{g} \mathrm{g}^{-1}$ para F. Aysén).

Se calculó el índice de geoacumulación (Igeo) para el Zn utilizando la relación Igeo $=\log _{2}(\mathrm{Cn} / 1,5 \mathrm{Bn})$, donde $\mathrm{Cn}$ es la concentración de $\mathrm{Zn}$ experimental obtenida en este estudio en $\mu \mathrm{g} \mathrm{g}^{-1}$ y Bn el valor geoquímico de background que correspondió a la línea base de $\mathrm{Zn}=84 \mu \mathrm{g} \mathrm{g}^{-1}$.

\section{Tratamiento DE LOS DATOS}

La caracterización del sedimento se realizó aplicando a los datos estadística descriptiva con el programa computacional Statistica versión 6.0 (StatSoft. Inc. 2001). Se agrupó la información de acuerdo a datos que resultaron homogéneos y se separó los valores heterogéneos mediante análisis de Box plot, utilizando como criterio 3 desviaciones estándar y $15 \%$ de coeficiente de variación. Se aplicó ANOVA y una prueba a posteriori, para visualizar posibles diferencias entre las medias con $\alpha$ de 0,05 .

\section{Resultados}

Los sedimentos al interior del Fiordo Aysén presentaron una textura homogénea, i.e., limo arcilla, con un tamaño de grano inferior a $0,65 \mu \mathrm{m}$. El contenido de materia orgánica en los sedimentos superficiales fue mayor en la cabeza del fiordo, con un valor de 9,5 $\pm 1,2 \%$ (Est. 82), disminuyendo hacia la boca del fiordo para alcanzar un valor de 3,7 $\pm 0,4 \%$ (Est. 77) (Tabla 2).

El contenido de materia orgánica total (\%), no mostró diferencias significativas entre estaciones contiguas, pero si se observó diferencias significativas en el contenido de materia orgánica total entre las estaciones extremas i.e., Est. 82 (cabeza del fiordo) y Est. 77 (boca del fiordo), (ANOVA, $\left.\mathrm{F}_{(5,57)}=10,62 ; P=0,001\right)$. Los coeficientes de variación del contenido de materia orgánica total en el sedimento superficial, fueron mayores en la parte media del fiordo y hacia la cabeza, Ests. 79, 81 y 82 (Tabla 2).

La concentración de $\mathrm{Zn}$ total medida en las muestras de las fracciones (profundidad del sedimento), en las 5 estaciones del fiordo, se muestran en la Tabla 3. La concentración de $\mathrm{Zn}$ total promedio en el sedimento superficial del fiordo, fluctuó entre 78,8 $\mathrm{gg} \mathrm{g}^{-1}$ (boca del fiordo) y $138,7 \mu \mathrm{g} \mathrm{g}^{-1}$ (cabeza del fiordo) con diferencias
Tabla 2. Materia orgánica total promedio (\%) en los sedimentos de cada una de las estaciones muestreadas en el Fiordo Aysén. Crucero CIM AR 13-FIORDO (n=3), noviembre 2007 / Average total organic matter (\%) in the sediment of the stations sampled in the Aysén Fjord. CIMAR 13-FJORD, $(n=3)$, November 2007

\begin{tabular}{crrrrr}
\hline & \multicolumn{5}{c}{ Estaciones Fiordo Aysén } \\
\cline { 2 - 6 } & 77 & 78 & 79 & 81 & 82 \\
\hline Promedio & 3,7 & 5,4 & 5,9 & 6,2 & 9,5 \\
DS & 0,4 & 0,7 & 1,3 & 1,5 & 1,2 \\
CV & 9,9 & 6,2 & 17,5 & 20,3 & 15,8 \\
\hline
\end{tabular}

DS= desviación estándar; $\mathrm{CV}=$ coeficiente de variación

Tabla 3. Contenido de Zn promedio en sedimentos del Fiordo Aysén $(n=6)$. Crucero CIMAR 13-FIORDO, noviembre 2007 / Average of $\mathrm{Zn}$ content in the Aysén Fjord sediments $(n=6)$. CIMAR 13-FJORD, November 2007

\begin{tabular}{|c|c|c|c|c|c|c|}
\hline \multirow{2}{*}{$\begin{array}{l}\text { Estratos } \\
\quad(\mathrm{cm})\end{array}$} & \multirow[t]{2}{*}{ Estadísticas } & \multicolumn{5}{|c|}{$\begin{array}{l}\text { Contenido de } \mathrm{Zn}\left(\mu \mathrm{g} \mathrm{g}^{-1}\right) \\
\text { en estaciones Fiordo Aysén }\end{array}$} \\
\hline & & Est. 77 & Est. 78 & Est. 79 & Est. 81 & Est. 82 \\
\hline \multirow[t]{3}{*}{$(0-2)$} & Promedio & 78,8 & 118,5 & 84,9 & 130,3 & 138,7 \\
\hline & DS & 9,3 & 40,3 & 7,2 & 34,7 & 20,8 \\
\hline & $\mathrm{CV}$ & 11,6 & 34,1 & 8,5 & 26,7 & 15,1 \\
\hline \multirow[t]{3}{*}{$(4-5)$} & Promedio & 82,5 & 91,0 & 67,8 & 146,7 & 188,3 \\
\hline & DS & 10,8 & 15,7 & 8,8 & 74,2 & 28,2 \\
\hline & $\mathrm{CV}$ & 12,3 & 16,2 & 12,9 & 50,6 & 15,0 \\
\hline \multirow[t]{3}{*}{$(6-7)$} & Promedio & 93,8 & 97,5 & 90,8 & 122,9 & 109,3 \\
\hline & DS & 10,1 & 14,7 & 9,9 & 13,1 & 15,9 \\
\hline & $\mathrm{CV}$ & 11,1 & 15,1 & 10,9 & 10,7 & 14,5 \\
\hline \multirow[t]{3}{*}{$(10-11)$} & Promedio & 89,2 & 84,7 & 85,7 & 152,8 & 177,5 \\
\hline & DS & 14,7 & 2,7 & 11,5 & 1,2 & 25,1 \\
\hline & $\mathrm{CV}$ & 16,5 & 3,2 & 13,4 & 0,8 & 14,1 \\
\hline \multirow[t]{3}{*}{$(15-16)$} & Promedio & $\mathrm{S} / \mathrm{M}$ & 72,7 & 111,0 & 102,8 & 92,3 \\
\hline & DS & & 21,0 & 67,8 & 2,8 & 15,0 \\
\hline & $\mathrm{CV}$ & & 28,9 & 61,1 & 2,8 & 16,3 \\
\hline \multirow[t]{3}{*}{$(20-21)$} & Promedio & $\mathrm{S} / \mathrm{M}$ & 74,6 & 94,9 & 89,1 & 86,5 \\
\hline & DS & & 6,8 & 2,0 & 0,8 & 1,8 \\
\hline & $\mathrm{CV}$ & & 9,1 & 2,2 & 0,9 & 2,1 \\
\hline Promedio total & & 86,1 & 89,8 & 89,2 & 124,1 & 132,0 \\
\hline
\end{tabular}

$\mathrm{CV}=$ coeficiente de variación. $\mathrm{DS}=$ desviación estándar. $\mathrm{S} / \mathrm{M}=$ Sin muestra

significativas entre las estaciones analizadas (ANOVA, $\left.\mathrm{F}_{(4,68)}=17,92 ; P=0,0001\right)$. Las concentraciones de $\mathrm{Zn}$ en las estaciones de la cabeza del fiordo i.e., Est. 81 y Est. 82 fueron estadísticamente diferentes (prueba a posteriori). Sin embargo, los estratos en conjunto no presentaron diferencias significativas (ANOVA, $\mathrm{F}_{(5,68)}=1,81 ; P=0,122$ ). 
Se observó fluctuaciones en la concentración promedio de $\mathrm{Zn}$ total, entre los estratos de sedimento. Por ejemplo, en la Est. 79, en superficie la concentración de $\mathrm{Zn}$ fue de $84,9 \mu \mathrm{g} \mathrm{g}^{-1}$, inferior a sus vecinas, i.e., $180,3 \mu \mathrm{g} \mathrm{g}^{-1}$ (Est. 81) y $118,8 \mu \mathrm{g} \mathrm{g}^{-1}$ (Est. 78); en el estrato de profundidad de 4$5 \mathrm{~cm}$ la concentración de Zn total bajó hasta $67,8 \mu \mathrm{g} \mathrm{g}^{-1}$ (bajo el valor de línea base, $84-86 \mu \mathrm{g} \mathrm{g}^{-1}$ ) e inferior a sus vecinas, i.e., $81 \mu \mathrm{g} \mathrm{g}^{-1}$ (Est. 78) y 146,7 $\mu \mathrm{g} \mathrm{g}^{-1}$ (Est. 81), aumentando la concentración en el mismo estrato hasta $188,3 \mu \mathrm{g} \mathrm{g}^{-1}$ (Est.82), la estación más cercana a Puerto Chacabuco. Sin embargo, la concentración media de $\mathrm{Zn}$ no mostró diferencias estadísticamente significativas entre estaciones vecinas (Fig. 2), observándose bajo los $10 \mathrm{~cm}$ de profundidad del sedimento en las Ests. 77, $78 \mathrm{y}$ 79 los valores más cercanos a los de línea base, i.e., 84 $86 \mu \mathrm{g} \mathrm{g}^{-1}$ (Tabla 3).

Al realizar el análisis por estación, las estaciones 79 y 81 ubicadas en el sector cercano en que se produjeron las avalanchas, no presentaron diferencias significativas entre los estratos, i.e., estación 77 (ANOVA, $\mathrm{F}_{(11,3)}=2,06$; $P=0,184$ ), estación 78 (ANOVA, $\mathrm{F}_{(5,17)}=1,59, P=0,2408$ ); estación 79 (ANOVA, $\mathrm{F}_{(5,17)}=1,45 ; P=0,2763$ ), estación 81 (ANOVA, $\mathrm{F}_{(5,17)}=3,19 ; P=0,0464$ ), estación 82 (ANOVA, $\left.\mathrm{F}_{(11,3)}=3,01 ; P=0,99\right)$, observándose una mayor variabilidad en la concentración de Zn en el sector de la cabeza del fiordo.

\section{Discusión}

El patrón de comportamiento del contenido de $\mathrm{Zn}$ en los sedimentos del Fiordo Aysén propuesto por Ahumada (1998) y Ahumada et al. (2004, 2007) presentaba 2 características, un gradiente horizontal en los sedimentos superficiales que disminuía hacia la boca, i.e., Est. de la cabeza del fiordo con $181 \pm 29 \mu \mathrm{g} \mathrm{g}^{-1}$ (Ahumada 1998) y $168 \pm 15 \mu \mathrm{g} \mathrm{g}^{-1}$ (Ahumada et al. 2004), y Est. de la boca con $85,8 \pm 4 \mu \mathrm{g} \mathrm{g}^{-1}$ (Ahumada 1998) y 83,6 $46 \mu \mathrm{g} \mathrm{g}^{-1}$ (Ahumada et al. 2004) y una zona de enriquecimiento superficial en la distribución vertical de los primeros 10 cm en la cabeza del fiordo que se estabilizaba después de los $15 \mathrm{~cm}$, con concentraciones que se acercaban a la de línea base, i.e., $84-86 \mu \mathrm{g} \mathrm{g}^{-1}$.

En este estudio, se observó que el gradiente superficial en la concentración de $\mathrm{Zn}$ total desde la cabeza a la boca se había alterado, con concentraciones superficiales menores en las Ests. 77 y 79, que no fueron estadísticamente significativas pero sí con una alta desviación estándar y coeficiente de variación (Tabla 3).

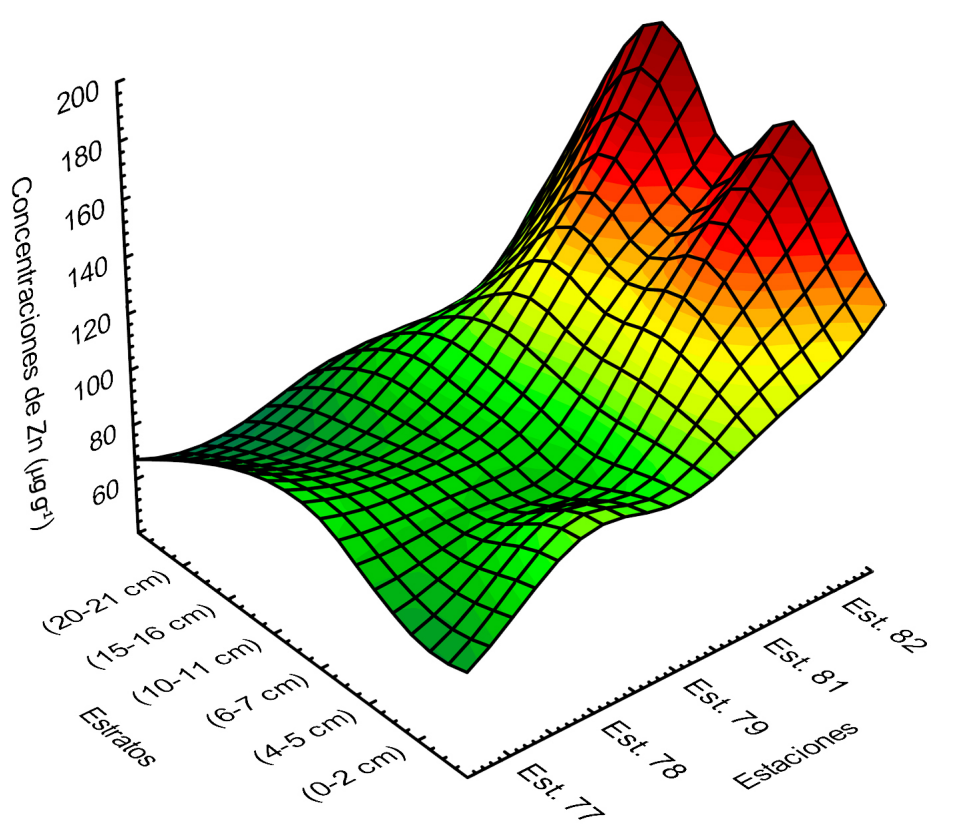

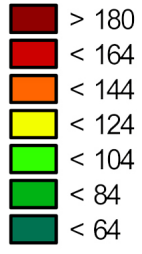

Figura 2. Concentraciones de $\mathrm{Zn}$ total, en los estratos de sedimento analizados, en las diferentes estaciones. CIMAR 13-FIORDO, noviembre 2007 / Total Zn concentration of sediment layers, in a core at the sites of Aysén Fjords. CIMAR 13-FJORD, in November 2007 
Si se toma los altos CV como una evidencia de redistribución vertical de $\mathrm{Zn}$ en el sedimento (sedimento no homogéneo), se podría considerar que la actividad sísmica y posterior tsunami, afectó la columna de sedimentos. Por ejemplo, en la Est. 81, se observó un CV de $26,7 \%$ en superficie $(0-2 \mathrm{~cm})$ y de $50,6 \%$ a una profundidad de $4-5 \mathrm{~cm}$. Del mismo modo, en la Est. 79 se observó un CV de 61,1\% a una profundidad de $15-16 \mathrm{~cm}$, lo que correspondería a la mayor profundidad afectada y que coincide con la estación más cercana a la Falla de Liquiñe-Ofqui y de la zona de avalanchas (Tabla 3). Sin embargo, en estaciones con profundidades mayores de $100 \mathrm{~m}$ (columna de agua) la resuspensión del sedimento fino del fondo pareciera poco probable, excepto por la acción de forzantes sísmicos, como los que se produjeron en el Fiordo Aysén, con cuencas > 100 m de profundidad.

Del mismo modo: los altos CV indicarían que los sedimentos al momento de muestreo, no se encontraban aun completamente asentados, ya que la estructura vertical del sedimento habría sido afectada (Tabla 3). Otra alteración importante fue la disminución de la concentración de $\mathrm{Zn}$ en la cabeza del fiordo (Est. 82) con 138,7 $\mu \mathrm{g} \mathrm{g}^{-1}$, menor que en los cruceros anteriores [i.e., $168 \pm 15$ (CIMAR 7-Fiordo) y $181 \pm 29 \mu \mathrm{g} \mathrm{g}^{-1}$ (CIMAR 1-Fiordo)], y además, en la boca (Est. 77 con $78,8 \mu \mathrm{g} \mathrm{g}^{-1}$ ) concentración de Zn menor que la línea base $\left(84-86 \mu \mathrm{g} \mathrm{g}^{-1}\right)$.

Los índices analizados, factor de enriquecimiento (FE) e índice de geoaccumulación (Igeo) fueron calculados con el objeto de detectar anomalías en la redistribución de $\mathrm{Zn}$ a lo largo del fiordo (Tabla 4). Según Zhang \& Liu (2002), factores de enriquecimiento entre 0,8 y 1,5 indicarían que el metal sería aportado principalmente por procesos naturales, como erosión de rocas madres y materiales de la corteza, mientras que factores de enriquecimiento mayores a 1,5 sugerirían enriquecimiento por redistribución de sedimentos por procesos sedimentarios. Del mismo modo, Birch et al. (2001) postulan que $\mathrm{FE}<2$ en un área, no corresponderían necesariamente a un proceso de enriquecimiento, sino más bien a la heterogeneidad y variabilidad del medio. En este contexto, los factores de enriquecimiento estimados para el Fiordo Aysén indicarían que se está en presencia de un área 'de no contaminada a moderadamente contaminada', aplicando los conceptos de FE e Igeo (Tablas 5 y 6). Sólo la Est. 82 mostró un índice $\geq$ 2 indicando un aporte antropogénico de $\mathrm{Zn}$ en los sedimentos, situación que no es distinta de lo observado en los trabajos anteriores (Ahumada 1998, Ahumada et al. 2004, 2007); lo que sugiere que el bajo enriquecimiento antropogénico de $\mathrm{Zn}$ total en el sector de la cabeza del
Tabla 4. Factor de enriquecimiento (FE) e índice de geoacumulación (Igeo) respecto de la profundidad del sedimento, estimado en el Fiordo Aysén. Crucero CIMAR 13-FIORDO, noviembre 2007 / Enrichment factor (EF) and geo-accumulation index (Igeo) respect on the depth of the sediment, estimated at Aysén Fjord. CIMAR 13-FJORD, November 2007

\begin{tabular}{|c|c|c|c|c|c|}
\hline $\begin{array}{l}\text { Est } \\
\mathrm{N}^{0 .}\end{array}$ & $\begin{array}{l}\text { Prof. } \\
\text { Z (cm) }\end{array}$ & $\begin{array}{l}\text { Valor Exp. } \\
\text { Zn }\left(\mu \mathrm{g} \mathrm{g}^{-1}\right)\end{array}$ & $\begin{array}{l}\text { Valor Exp. } \\
\operatorname{Li}\left(\mu g^{-1}\right)\end{array}$ & $\begin{array}{c}\text { Igeo } \\
\text { Zn Igeo }\end{array}$ & $\begin{array}{c}\mathrm{FE} \\
\text { EF Zn }\end{array}$ \\
\hline \multirow[t]{4}{*}{77} & $(0-2)$ & 78,8 & 27,0 & 0,96 & 0,9 \\
\hline & $(4-5)$ & 88,1 & 27,7 & 1,08 & 1,0 \\
\hline & $(6-7)$ & 91,5 & 22,5 & 1,44 & 1,3 \\
\hline & $(10-11)$ & 89,2 & 20,8 & 1,52 & 1,4 \\
\hline \multirow[t]{6}{*}{78} & $(0-2)$ & 118,5 & 28,5 & 1,47 & 1,3 \\
\hline & $(4-5)$ & 97,0 & 30,2 & 1,1 & 1,0 \\
\hline & $(6-7)$ & 97,5 & 26,7 & 1,28 & 1,2 \\
\hline & $(10-11)$ & 84,7 & 22,4 & 1,33 & 1,2 \\
\hline & $(15-16)$ & 72,7 & 21,1 & 1,2 & 1,1 \\
\hline & $(20-21)$ & 74,6 & 22,3 & 1,16 & 1,1 \\
\hline \multirow[t]{6}{*}{79} & $(0-2)$ & 84,9 & 36,4 & 0,64 & 0,8 \\
\hline & $(4-5)$ & 67,8 & 26,1 & 0,79 & 0,8 \\
\hline & $(6-7)$ & 90,8 & 31,7 & 0,93 & 0,9 \\
\hline & $(10-11)$ & 85,7 & 27,2 & 1,07 & 1,0 \\
\hline & $(15-16)$ & 111,0 & 25,6 & 1,53 & 1,4 \\
\hline & $(20-21)$ & 94,9 & 24,8 & 1,35 & 1,2 \\
\hline \multirow[t]{6}{*}{81} & $(0-2)$ & 130,3 & 29,4 & 1,56 & 1,4 \\
\hline & (4-5) & 146,7 & 31,5 & 1,63 & 1,5 \\
\hline & $(6-7)$ & 122,9 & 28,2 & 1,54 & 1,4 \\
\hline & $(10-11)$ & 152,8 & 26,9 & 1,92 & 1,8 \\
\hline & $(15-16)$ & 102,8 & 26,5 & 1,37 & 1,3 \\
\hline & $(20-21)$ & 89,1 & 24,8 & 1,26 & 1,2 \\
\hline \multirow[t]{6}{*}{82} & $(0-2)$ & 138,7 & 22,5 & 2,04 & 2,0 \\
\hline & $(4-5)$ & 188,3 & 24,7 & 2,35 & 2,5 \\
\hline & (6-7) & 109,3 & 35,2 & 1,05 & 1,0 \\
\hline & $(10-11)$ & 177,5 & 26,1 & 2,18 & 2,2 \\
\hline & $(15-16)$ & 92,3 & 24,8 & 1,31 & 1,2 \\
\hline & $(20-21)$ & 86,5 & 25,3 & 1,19 & 1,1 \\
\hline
\end{tabular}

fiordo, es una de las condiciones que se mantuvo en el fiordo.

Dos elementos son importantes en el análisis de este escenario: i) la disminución de la concentración de $\mathrm{Zn}$ en la superficie de la Est. 82 e índices bajos, y ii) el incremento de la concentración de Zn e índices mayores en los estratos inmediatamente inferiores. Ambos indicarían que el efecto de los forzantes fue local y podría haber 
Tabla 5. Escala y conceptos usados para interpretar los valores de concentración de $\mathrm{Zn}$ total obtenidos de la aplicación del factor de enriquecimiento (FE) / Scale and conceps values, used to interpret the total $\mathrm{Zn}$ concentration resulting from the application of the enrichment factor (EF)

\begin{tabular}{ll}
\hline Intervalos de $\mathrm{FE}$ & Fuente $\mathrm{u}$ origen del metal \\
\hline $0,0<\mathrm{FE} \leq 1,5$ & Producto de erosión de la roca madre. \\
$1,5<\mathrm{FE} \leq 3,0$ & $\begin{array}{l}\text { Enriquecido por procesos sedimentarios: sedimentación } \\
\text { fina, materia orgánica o precipitados inorgánicos. }\end{array}$ \\
$3,0<\mathrm{FE} \leq 6,2$ & Valores de enriquecimiento por fuentes externas. \\
$\mathrm{FE}>6,2$ & Contaminación por fuentes antrópicas.
\end{tabular}

Fuente: Modificado de Lawson \& Winchester (1979)

Tabla 6. Escala y conceptos usados para interpretar los valores obtenidos del índice de geo-acumulación / Scale and concepts used to evaluate the results of Geoaccumulation Index

\begin{tabular}{cll}
\hline $\begin{array}{c}\text { Escala } \\
\text { Igeo }\end{array}$ & \multicolumn{1}{c}{$\begin{array}{c}\text { Intervalos } \\
\text { Igeo }\end{array}$} & \multicolumn{1}{c}{ Conceptos } \\
\hline 0 & Igeo $\leq 0,0$ & Prístino a no alterado \\
1 & $0,0<$ Igeo $\leq 1,1$ & No contaminado a levemente alterado \\
2 & $1,1<$ Igeo $\leq 2,1$ & Levemente a moderadamente contaminado \\
3 & $2,1<$ Igeo $\leq 3,1$ & Moderadamente a fuertemente contaminado \\
4 & $3,1<$ Igeo $\leq 4,1$ & Fuertemente a extremadamente contaminado \\
5 & $4,1<$ Igeo $\leq 5,0$ & Extremadamente contaminado \\
6 & Igeo $>5,0$ & Requiere estudios de remediación \\
\hline
\end{tabular}

alcanzado hasta los $10 \mathrm{~cm}$ en la columna de sedimentos, produciendo cambios menores en la concentración de Zn, debido a una sedimentación diferenciada de las partículas, con la excepción de lo observado en la Est. 79 (CV de 61,1\%). Además, sería posible que el efecto local observado, se deba a las condicionantes físicas que imponen los desniveles o cubetas a lo largo del fiordo, lo que limitaría la redistribución del sedimento. Por lo que, el forzante físico pudo producir una resuspensión y resedimentación en las mismas cubetas sin transporte de material más allá de ellas, lo que explicaría las variaciones verticales en la concentración de Zn (Tabla 3). Por su parte, el desplazamiento de material resuspendido ocurriría en las partes del fiordo con menor profundidad, esto es, la cabeza del fiordo ( $c a$., $100 \mathrm{~m}$ de profundidad). Finalmente, se sugiere que el efecto producido por las avalanchas de material desde las costas escarpadas mostraría su efecto en las Est. 79 y 81, generando en estos sectores la variabilidad que se observa en la mayor parte de la columna de sedimentos.
El posterior tsunami que generó ondas superficiales locales, a pesar de la energía liberada, no habría logrado producir un cambio en el patrón de distribución general de los sedimentos del fiordo y al parecer tampoco fue un factor significativo en la movilización de los sedimentos, ya que no se observó variaciones importantes en la granulometría, por lo que se infiere que el material que ingresó por las avalanchas fue un fenómeno puntual, que removió el sedimento sólo en los bordes. El material terrígeno de las avalanchas, no fue detectado en las estaciones estudiadas, salvo en la Est. 79 entre 4-5 cm de profundidad, donde la concentración de $\mathrm{Zn}$ disminuyó bajo los niveles de línea base, i.e., $67,8 \mu \mathrm{g} \mathrm{g}^{-1}$.

Se concluye que, cambios leves se detectaron en la mitad posterior del fiordo, donde los valores de concentración de $\mathrm{Zn}$ total superficial se hicieron homogéneos, pero las concentraciones de $\mathrm{Zn}$ de la cabeza fueron más bajas que las registradas en los cruceros anteriores. Finalmente, el efecto de los forzantes fue local y superficial, i.e., en el sedimento los cambios no excedieron los 10 primeros $\mathrm{cm}$ de la columna de sedimentos y la Est. 79 fue la que mostró un mayor efecto.

\section{Agradecimientos}

Se agradece al Ministerio de Hacienda y Comité Oceanográfico Nacional (CONA-Chile) por el financiamiento del Proyecto CIMAR13FIORDO 07-09 y por la Beca CONA CIMAR otorgada a Mauricio Garrido para su titulación de Químico Marino. Esta es una contribución al Proyecto asignado al Dr. Ramón Ahumada B. Se agradece además, a la Dirección de Investigación de la Universidad Católica de la Santísima Concepción por la gestión y apoyo y al Comandante y tripulación del 'AGOR Vidal Gormaz' por la asistencia a bordo durante la fase de muestreo. A la Srta. Emma Cascales por la recolección de muestras durante el crucero. A Sra. Vanessa Novoa por los análisis estadísticos

\section{LITERATURA CITADA}

Ahumada R. 1998. Metales traza contenidos en los sedimentos del Seno Aysén: Línea base y alteraciones antrópicas. Ciencia y Tecnología del Mar 21: 75-88.

Ahumada R, E González \& JY Neira. 2004. Especiación de zinc en sedimentos marinos del fiordo Aysén. Investigaciones Marinas 32(1): 3-10.

Ahumada R, E González, V Medina \& A Rudolph. 2007. Análisis de las principales fuentes de cinc en sedimentos marinos de la zona estuarial del Fiordo Aysén. Investigaciones Marinas 35(2): 5-14. 
Aloupi M \& MO Angelidis. 2001. Normalization to lithium for the assessment of metal contamination in coastal sediment cores from the Aegean Sea, Greece. Marine Environmental Research 52: 1-12.

Antezana T. 1999. Hydrographic feature of Magellan and Fueguian in land passages and adjacent subantarctic waters. Scientia Marina 63(Suppl.1): 69-80.

Araya-Vergara JF. 1997. Perfiles geomorfológicos de los fiordos y depresión longitudinal de Norpatagonia. Ciencia y Tecnología del Mar 20: 3-22.

Birch G, M Siaka \& C Owens. 2001. The sources of anthropogenic heavy metals in fluvial sediments of rural catchment Coxs River, Australia. Water, Air \& Soil Pollution 126(1-2): 13-35.

Clapperton C M. 1994. The quaternary glaciation of Chile: a review. Revista de Historia Natural 67: 369-383.

Delgado DS. 2004. Relación entre el perfil del basamento en fiordos y canales y la morfoestructura regional en norpatagonia. Tesis de Magíster en Geografía, mención Evaluación Territorial, Universidad de Chile, Santiago, 85 pp.

Lawson DR \& JW Winchester. 1979. A standard crustal aerosol as a reference for elemental enrichment factors. Atmospheric Environment 10: 925-930.

Luczak C, M Janquin \& A Kupka. 1997. Simple standard procedure for the routine determination of organic matter in marine sediment. Hydrobiologia 345: 87-94.

Marín VH, LE Delgado \& A Tironi. 2008. The Aysén fjord tsunami of April 2007: unexpected uses of circulation models. In: Neves RJ, N Baretta \& M Mateus (eds). Perspectives on integrated Coastal Zone Management in South America, pp. 597-601. IST Press, Lisboa.
Müller G. 1969. Index of geoaccumulation in sediments of the Rhine River. Geological Journal 2: 109-118.

Pickard G. 1971. Some physical oceanographic features of inlets of Chile. Journal of the Fisheries Research Board of Canada 28: 1077-1106.

Salamanca M. 1996. Geocronología de sedimentos marinos de la zona de fiordos de la XIRegión. Informes Preliminares, Crucero CIMAR1 FIORDO, pp. 64-68. Servicio Hidrográfico y Oceanográfico de la Armada de Chile, Valparaíso.

Sepúlveda SA \& A Serey. 2009. Tsunamigenic, earthquaketriggered rock slope failures during the April 21, 2007 Aisén earthquake, southern Chile (45.5 $\left.{ }^{\circ} \mathrm{S}\right)$ Andean Geology 36(1): 131-136.

Silva N \& D Guzmán. 2006. Condiciones oceanográficas físicas y químicas, entre Boca del Guafo y Fiordo Aysén (Crucero CIMAR7 FIORDO). Ciencia y Tecnología del Mar 29(1): 25-44.

Silva N, C Calvete \& H Sievers. 1997. Características oceanográficas físicas y químicas de canales australes chilenos entre Puerto Montt y laguna San Rafael (Crucero CIMAR1 FIORDO). Ciencia y Tecnología del Mar 20: 23 106.

Zhang J \& CL Liu. 2002. Riverine composition and estuarine geochemistry of particulate metals in China - Weathering features, anthropogenic impact and chemical fluxes. Estuarine Coastal and Shelf Science 54: 1051-1070. 